\title{
Interactive comment on "High-precision
} atmospheric oxygen measurement comparisons between a newly built CRDS analyzer and existing measurement techniques" by Tesfaye A. Berhanu \section{et al.}

\section{Tesfaye A. Berhanu et al.}

tberhanu@picarro.com

Received and published: 13 May 2019

We have provided our replies as a supplement

Please also note the supplement to this comment:

https://www.atmos-meas-tech-discuss.net/amt-2018-396/amt-2018-396-AC1-

Interactive comment on Atmos. Meas. Tech. Discuss., doi:10.5194/amt-2018-396, 2019. 